\title{
LINC00271 inhibits epithelial-mesenchymal transition of papillary thyroid cancer cells by downregulating trefoil factor 3 expression
}

\author{
Pihong Li ${ }^{\mathrm{a}, \#}$, Xiaoyu Pan, ${ }^{\mathrm{a}, \#}$, Zhouci Zheng ${ }^{\mathrm{a}}$, Yihan Sun $^{\mathrm{a}}$, Yifan Han ${ }^{\mathrm{a}}$, Jianda Dong $^{\mathrm{a},{ }^{*}}$ \\ ${ }^{a}$ Department of General Surgery, The Second Affiliated Hospital of Wenzhou Medical University, Wenzhou 325027, China.
}

\begin{abstract}
Objective: As an essential promoter of papillary thyroid cancer (PTC) metastasis, epithelial-mesenchymal transition (EMT) plays an important role in PTC progression. However, its upstream regulation mechanism is unknown. This study aimed to clarify the specific regulation mechanism of LINC00271 on the EMT of PTC cells. Methods: LINC00271 expression was detected in PTC tissues and PTC cell line TPC-1. The invasive and migratory capabilities of TPC-1 cells were determined by Transwell invasion and wound-healing assays, respectively. The interaction between LINC00271 and trefoil factor 3 (TFF3) was confirmed with RNA pull-down and RNA immunoprecipitation assays. The stability of TFF3 was measured by cycloheximide-chase and ubiquitination assays.

Results: LINC00271 was downregulated in both tumor tissue of PTC patients and PTC cell line TPC-1. Its expression inversely correlated with the expression of TFF3. Functionally, LINC00271 overexpression inhibited the EMT of TPC- 1 cells and reduced the tumor volumes in xenograft model mice. Further investigation confirmed that LINC00271 bound to TFF3 and suppressed its expression by increasing its ubiquitination level. Moreover, TFF3 overexpression abrogated the inhibitory effect of LINC00271 on the EMT of TPC-1 cells.

Conclusion: LINC00271 inhibits the EMT of TPC-1 cells by downregulating TFF3 expression.

Keywords: LINC00271, trefoil factor 3, epithelial-mesenchymal transition, papillary thyroid cancer
\end{abstract}

\section{Introduction}

Papillary thyroid cancer (PTC) is the most common type of thyroid cancer, accounting for about $85 \%$ of all types [1]. Although most PTC patients tend to have good prognoses after clinical treatment, $30 \%$ are diagnosed with metastasis and die in a short time [2]. Epithelial-mesenchymal transition (EMT) is thought to play an essential role in tumor metastasis [3]. During EMT, thyroid epithelial cells gradually change to mesenchymal cells, losing their differentiation capacity and increasing their invasion

\footnotetext{
\# These authors contributed equally.

* Corresponding author: Jianda Dong

Mailing address: Department of General Surgery, The Second Affiliated Hospital of Wenzhou Medical University, Wenzhou 325027, China.

E-mail: dongjianda1973@126.com

Received: 22 April 2020 / Accepted: 03 June 2020
}

and migration ability, thereby leading to metastasis [3]. Therefore, exploring the upstream regulation mechanism of EMT is vital for the clinical prevention of PTC metastasis.

Trefoil factor 3 (TFF3), a member of the trefoil factor family, is well known as an oncogene [4-6]. Krause et al. [7] measured the TFF3 levels in 150 thyroid specimens and found that TFF3 expression was significantly higher in thyroid malignancy than in benign thyroid nodules. In PTC cell line TPC-1, TFF3 promotes EMT via the MAPK/ ERK signaling pathway [8]. TFF3 knockdown can effectively inhibit EMT and repress cell migration and invasion [9]. Although it is known that TFF3 is a promoter of EMT in PTC, its upstream regulation mechanism remains unclear.

Long non-coding RNA (lncRNA) is a class of non-coding RNA longer than 200 nucleotides whose dysregulation has been associated with the development of various cancers [10]. Recently, increasing attention has been paid to the role of lncRNA in TPC. Ma et al. [11] screened 220 lncRNAs that were dysregulated in PTC patients. The authors found that LINC00271 was downregulated in 
PTC specimens. Low LINC00271 expression was an independent risk factor for PTC metastasis and recurrence, indicating that LINC00271 may participate in the EMT of PTC cells. In light of these studies' findings, this study aimed to explore the modulatory effects of LINC00271 on EMT in PTC and to provide an intervention target for the clinical prevention and treatment of PTC metastasis.

\section{Materials and methods}

\section{Sample collection}

Forty fresh PTC and paired adjacent tissue samples were collected from patients through surgery at the Second Affiliated Hospital of Wenzhou Medical University (China). All samples were frozen immediately after lesion resection and stored for subsequent experiments. All procedures were approved by the Ethics Committee of the Second Affiliated Hospital of Wenzhou Medical University. All patients signed informed consent forms.

\section{Cell culture and transfection}

Human normal thyroid epithelial cell line Nthy-ori 3-1 and PTC cell line TPC-1 were purchased from the Cell Bank of Type Culture Collection of the Chinese Academy of Sciences (China). Both cell lines were cultured in Dulbecco's modified Eagle medium (DMEM; Gibco, USA) containing 10\% fetal bovine serum (FBS; Gibco) with 5\% $\mathrm{CO}_{2}$ at $37^{\circ} \mathrm{C}$.

TPC-1 cells were cultured in 6-well plates at a concentration of $4 \times 10^{5}$ cells/well. When the cells had been cultured to $75 \%$ confluence, they were transfected with an RNAi vector (si-LINC00271), overexpression vectors (pcDNALINC00271 and pIRES2-TFF3), or corresponding negative controls (si-control, pcDNA, and pIRES2-EGFP) using Lipofectamine 2000 (Invitrogen, USA).

\section{Quantitative reverse transcription polymerase chain reaction}

TRIzol Reagent (Invitrogen, USA) was used to isolate total RNA from tumor tissue or cells. The samples' quality was evaluated by spectrophotometry, and high-quality samples $(1.8<$ OD260/280 < 2.0) were transcribed into complementary DNA (cDNA) using a one-step RNA reverse transcription kit (Hai Gene, China). Quantitative reverse transcription polymerase chain reaction (qRT-PCR) was performed in triplicate using a SYBR Green PCR kit (QIAGEN, Germany). Gene expressions were calculated using the $2^{-\triangle \Delta C T}$ method, and the relative expression of LINC00271 and TFF3 was normalized to $\beta$-actin. The sequences of the primers used in the experiments are shown in Table 1.

\section{Western blot analysis}

Total proteins from tumor tissue or cells or subcutaneous xenografts of mice were isolated using a radioimmunoprecipitation assay (RIPA) buffer (CoWin Biosciences,
Table 1. Sequences of the primers used in the experiments

\begin{tabular}{ll}
\hline qRT-PCR Primers & Sequences \\
\hline \multirow{2}{*}{ LINC00271 } & F 5'-GCTATTGGTGGGAGGCTTCAG-3' \\
& R 5'-TGGGCTGGACTTAATGACTTGC-3' \\
TFF3 & F 5'-CTTGCTGTCCTCCAGCTCT-3' \\
& R 5'- CCGGTTGTTGCACTCCTT-3' \\
\multirow{3}{*}{-actin } & F 5'-GCACTCTTCCAGCCTTCCTT-3' \\
& R 5'-TGGGCTGGACTTAATGACTTGC-3'
\end{tabular}

China). The protein was electrophoresed by $10 \%$ polyacrylamide gel electrophoresis (SDS-PAGE) and transferred to polyvinylidene fluoride membranes (Thermo Fisher Scientific, USA). The membranes were treated with 5\% skimmed milk for $30 \mathrm{~min}$ and incubated with primary anti-TFF3 (1:1000; Abcam, USA), anti-E-cadherin (1:300; Abcam), anti-zonula occluden-1 (1:500; Abcam), anti$\mathrm{N}$-cadherin (1:1000; Abcam), anti-fibronectin (1:1000; Abcam), and anti- $\beta$-actin (1:5000; Abcam) antibodies at $4{ }^{\circ} \mathrm{C}$ overnight. The next day, the membranes were incubated with Goat Anti-Rabbit IgG H\&L (1:2000; Abcam) for $2 \mathrm{~h}$. The bands were visualized using an iBright FL1500 Intelligent Imaging System (Thermo Fisher Scientific), and the protein levels were quantified using the ImageJ software.

\section{Transwell invasion assays}

Before the assays, Transwell chamber membranes (Corning, USA) were pre-coated using Matrigel (Corning). The upper chamber was filled with serum-free DMEM containing $1 \times 10^{3}$ TPC- 1 cells, and the lower chamber was filled with DMEM containing 10\% FBS. The chambers were then cultured in a $5 \% \mathrm{CO}_{2}$ incubator at $37^{\circ} \mathrm{C}$. Fortyeight hours later, invading cells on the lower side of the filter were stained using $0.5 \%$ crystal violet (CoWin Biosciences) and counted using a microscope.

\section{Wound-healing assays}

TPC-1 cells were seeded into 6-well plates. When they had grown to $90-95 \%$ confluence, the monolayer of the cells were scratched with a micropipette tip [12]. The wound areas were photographed at two time points ( 0 and $24 \mathrm{~h}$ ), and the distance between the groups was calculated. RNA immunoprecipitation assay

An RNA immunoprecipitation (RIP) assay kit (Magna RIP RNA-Binding Protein Immunoprecipitation Kit) was used according to the manufacturer's instructions (Merck Millipore, USA). TPC-1 cells were collected at 80-90\% confluence and lysed using $100 \mu$ of specific lysis buffer. Magnetic beads conjugated with human anti-TFF3 antibody were added into the lysate. The protein in the samples was removed with proteinase $\mathrm{K}$ to obtain the precipitated RNA, which was purified and used to detect LINC00271 by qRT-PCR. Immunoglobulin G (IgG) was used as a negative control.

\section{RNA pull-down assay}

Biotin-labeled LINC00271 was constructed with a Bio- 
tin RNA Labeling Mix (Roche, Switzerland) and T7 RNA polymerase (Roche). TPC-1 cells were collected at $80-90 \%$ confluence and lysed using $100 \mu \mathrm{l}$ of RIPA lysis buffer. The lysate was then incubated with biotin-labeled LINC00271 and streptavidin agarose magnetic beads at $4{ }^{\circ} \mathrm{C}$ for $1 \mathrm{~h}$. Western blotting was conducted to determine the expression of TFF3 in the LINC00271 pull-down complex.

\section{Cycloheximide-chase assay}

TPC-1 cells were transfected with pcDNA-LINC00271 or pcDNA. Forty-eight hours after transfection, the original medium was replaced with a fresh medium containing cycloheximide (CHX; $125 \mu \mathrm{g} / \mathrm{ml})$. The expression of TFF3 was then measured $0,1,2$, and $3 \mathrm{~h}$ after $\mathrm{CHX}$ treatment using western blot.

\section{Ubiquitination assay}

Flag-TFF3, HA-ubiquitin, and plasmid cloning DNA (pcDNA)-LINC00271 or pcDNA were co-transfected into TPC-1 cells. Forty-eight hours after transfection, the TPC-1 cells were incubated with MG132 (10 nM). Six hours later, total protein was isolated using RIPA. The cell lysates were then incubated with protein $A / G$ magnetic beads conjugated with anti-FLAG antibody overnight. Anti-ubiquitin antibody was used to determine the ubiquitination levels of TFF3.

Mouse xenograft models
Female BALB/c nude mice (4-6 weeks old) were purchased from the Institute of Laboratory Animal Sciences of the Chinese Academy of Medical Sciences. A lentiviral vector (LV)-LINC00271 and its negative control (LV-control) were obtained from RiboBio (China). For the establishment of the xenograft model, 12 mice were randomly divided into two groups. In the LV-LINC00271 group $(n=6), 1 \times 10^{6}$ TPC-1 cells transfected with LVLINC00271 were diluted in $100 \mu$ of DMEM and injected subcutaneously into the right flank of the mice. In the LVcontrol group $(n=6), 1 \times 10^{6}$ TPC- 1 cells transfected with LV-control were diluted in $100 \mu \mathrm{l}$ of DMEM and likewise injected into the mice. The tumor volumes in the mice were measured every seven days. After 28 days, the mice were sacrificed, and the tumor xenografts were collected for subsequent experiments. All experimental procedures were approved by the Ethics Committee of the Second Affiliated Hospital of Wenzhou Medical University.

\section{Statistical analysis}

All statistical analyses were performed using Prism 7.0 (GraphPad, USA). The experimental results were expressed as means \pm standard deviations (SD). Differences were assessed with Student's t-test or one-way analysis of variance (ANOVA) with the Newman-Keuls post-hoc test. The correlation between the expression of LINC00271 and TFF3 in PTC tissue samples was determined using the Pearson correlation coefficient. Results were considered statistically significant at a level of $\mathrm{P}<0.05$.
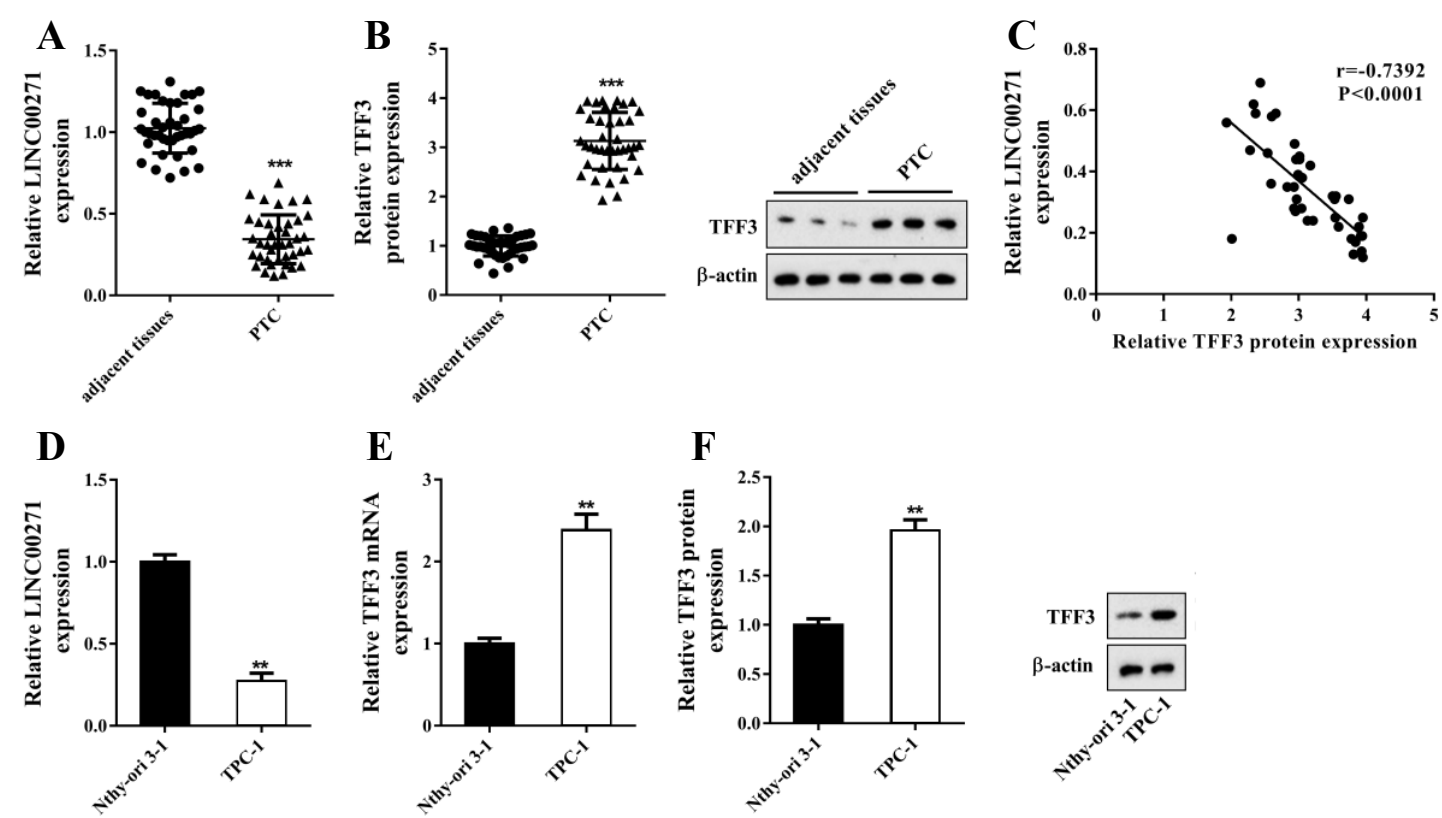

Figure 1. LINC00271 was downregulated in PTC tissue and a PTC cell line. Forty paired PTC and corresponding adjacent tissue samples were collected through surgery. A: LINC00271 expression measured by qRT-PCR; B: trefoil factor 3 (TFF3) expression measured by western blot and qRT-PCR; C: correlation plot of LINC00271 expression and TFF3 protein level. Higher TFF3 protein levels (x-axis) correlated with lower LINC00271 expression (y-axis). In normal thyroid epithelial cell line Nthy-ori 3-1 and PTC cell line TPC-1, D: LINC00271 expression measured by qRT-PCR; E: mRNA level of TFF3 measured by qRT-PCR; F: protein level of TFF3 measured by western blot. $* * \mathrm{P}<0.01, * * * \mathrm{P}<0.001$ vs. adjacent tissue or Nthy-ori 3-1. 


\section{Results}

LINC00271 was downregulated in PTC tissue and the PTC cell line

First, we detected the expression of LINC00271 in 40 paired PTC and corresponding adjacent tissue samples. The results showed that LINC00271 expression was markedly lower in PTC than in adjacent tissue (Figure 1A), indicating that LINC00271 may participate in PTC progression. Using online bioinformatics software, we identified four proteins (DiGeorge syndrome critical region 8 [DGCR8], forkhead box A1 [FOXA1], E2F transcription factor 4 [E2F4], and TFF3) that may interact with LINC00271 and have been proved associated with EMT [13-15]. Next, we overexpressed LINC00271 in PTC cell line TPC-1 and found that only TFF3 significantly decreased in response to LINC00271 overexpression

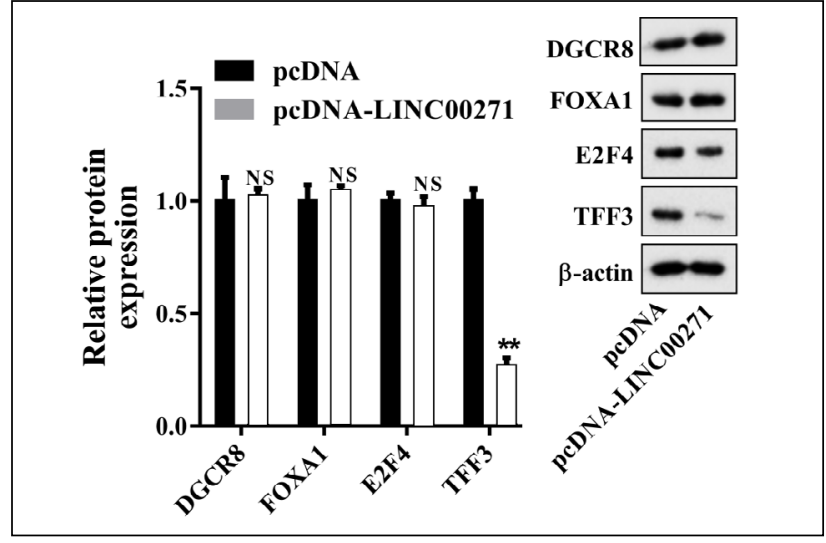

Supplemental Figure 1. Expression levels of DiGeorge syndrome critical region 8 (DGCR8), forkhead box A1 (FOXA1), E2F transcription factor 4 (E2F4), and TFF3 measured by qRT-PCR and western blot after transfection of TPC-1 cells with pcDNA-LINC00271 or pcDNA. ** $\mathrm{P}<$ 0.01 vs. pcDNA.

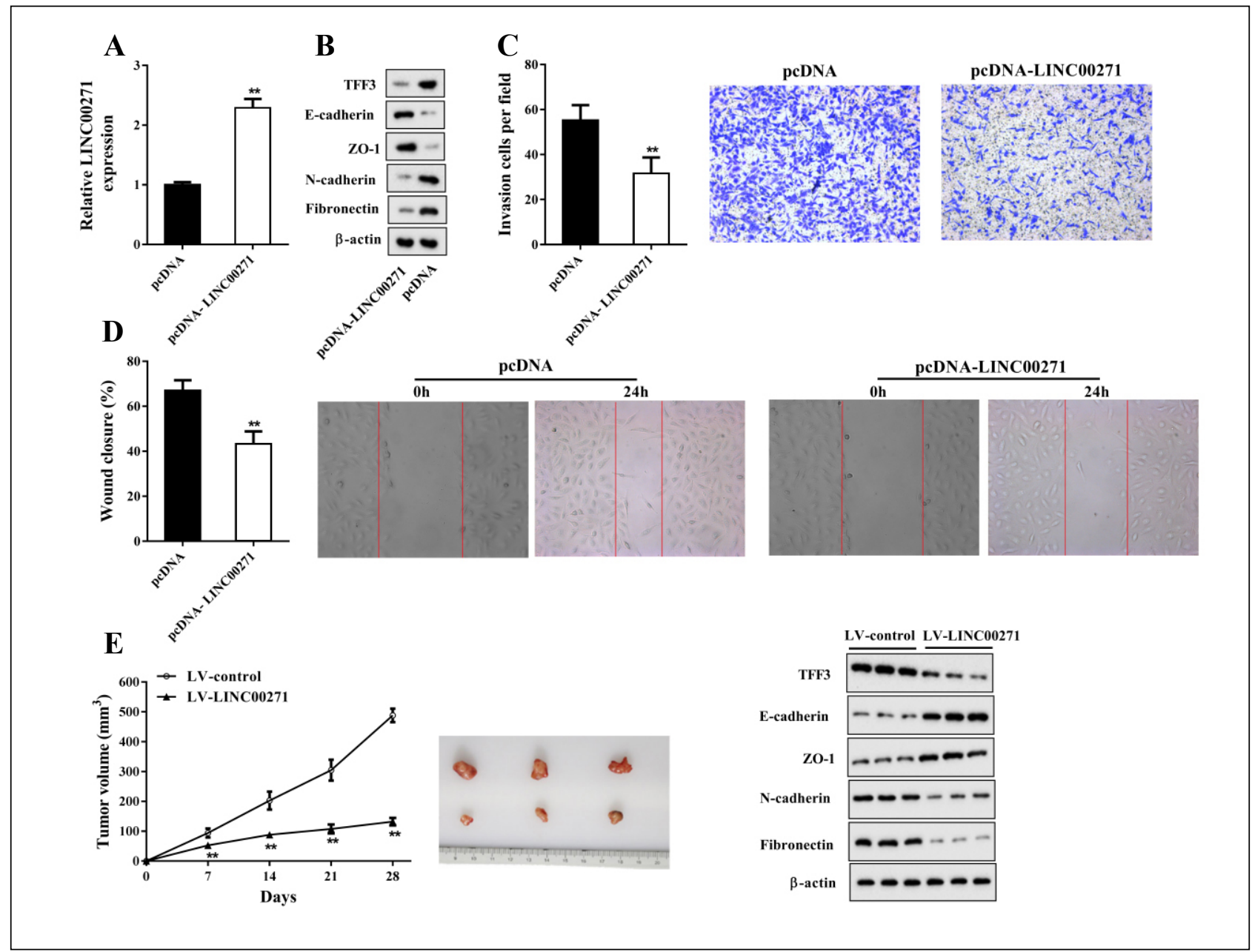

Figure 2. LINC00271 overexpression suppressed the EMT of PTC cells in vitro and in vivo. TPC-1 cells were transfected with pcDNA-LINC00271 or its negative control (pcDNA). Forty-eight hours later, the cells were harvested for experiments. A: LINC00271 expression measured by qRT-PCR; B: protein levels of TFF3, E-cadherin, zonula occluden-1 (ZO-1), N-cadherin, and fibronectin measured by western blot. Beta-actin was used as an internal control. C: Invasive capability of TPC-1 cells determined by Transwell invasion assay; D: migratory capability of TPC-1 cells determined by wound-healing assay. Xenograft model mice were divided into two groups: lentiviral vector $(\mathrm{LV})-\mathrm{LINC00271}(\mathrm{n}=6)$ and $\mathrm{LV}$-control $(\mathrm{n}=6)$. $\mathbf{E}$ : Tumor volumes of subcutaneous xenografts calculated 7, 14, 21, and 28 days after injection. F: Protein levels of TFF3, E-cadherin, ZO-1, N-cadherin, and fibronectin of subcutaneous xenografts measured by western blot. Beta-actin was used as an internal control. ** P $<0.01$ vs. pcDNA or LVcontrol. 
(Supplemental Figure 1). Therefore, we selected TFF3 for further experiments. The expression level of TFF3, measured by qRT-PCR and western blot (Figure 1B), showed a significant increase in PTC compared to adjacent tissue. A correlation plot further showed that the expression of LINC00271 inversely correlated with the expression of TFF3 in PTC tissue (Figure 1C). Moreover, in line with the results shown in Figure 1A and B, compared with normal thyroid epithelial cell line Nthy-ori 3-1, LINC00271 expression in PTC cell line TPC-1 decreased (Figure 1D), and TFF3 expression increased (Figure 1E and F).

\section{LINC00271 overexpression suppressed the EMT of PTC cells in vitro and in vivo}

To determine the role of LINC00271 in PTC, we overexpressed LINC00271 in PTC cell line TPC-1 using pcDNA-LINC00271 (Figure 2A). As shown in Figure 2B, after LINC00271 overexpression, the protein levels of TFF3 and mesenchymal markers N-cadherin and fibronectin [16] decreased, while those of epithelial markers E-cadherin and zonula occluden-1 (ZO-1) [17] increased. Transwell invasion assays (Figure 2C) and wound-healing assays (Figure 2D) showed that pcDNA-LINC00271 downregulated the invasive and migratory capabilities of TPC-1 cells. These findings indicate that LINC00271 upregula- tion suppressed the EMT of TPC-1 cells. Next, we validated our in vitro findings in $\mathrm{BALB} / \mathrm{c}$ nude mice. We found that compared to the LV-control group, LV-LINC00271 slowed the growth of tumor cells and noticeably reduced the tumor volumes (Figure 2E). Furthermore, as in the in vitro study, LINC00271 overexpression inhibited TFF3 expression and tumor cell EMT (Figure 2F).

\section{LINC00271 reduced TFF3 expression by weakening its stability}

In the previously described experiments, we found that LINC00271 expression inversely correlated with TFF3 expression in PTC tissue (Figure 1C) and that LINC00271 overexpression clearly suppressed the TFF3 protein levels in TPC-1 cells (Figure 2B). To examine whether there was an endogenous interaction between LINC00271 and TFF3 protein, we performed an RNA pull-down assay and RIP. As shown in Figure 3A, a large amount of TFF3 was detected in the complex pulled down by biotinylated LINC00271. Moreover, compared with IgG, a great quantity of LINC00271 was detected in the anti-TFF3 antibody precipitation complex (Figure 3B). Furthermore, TFF3 protein was downregulated in TPC-1 cells transfected with pcDNA-LINC00271 and upregulated in those transfected with si-LINC00271 (Figure 3C). In contrast,

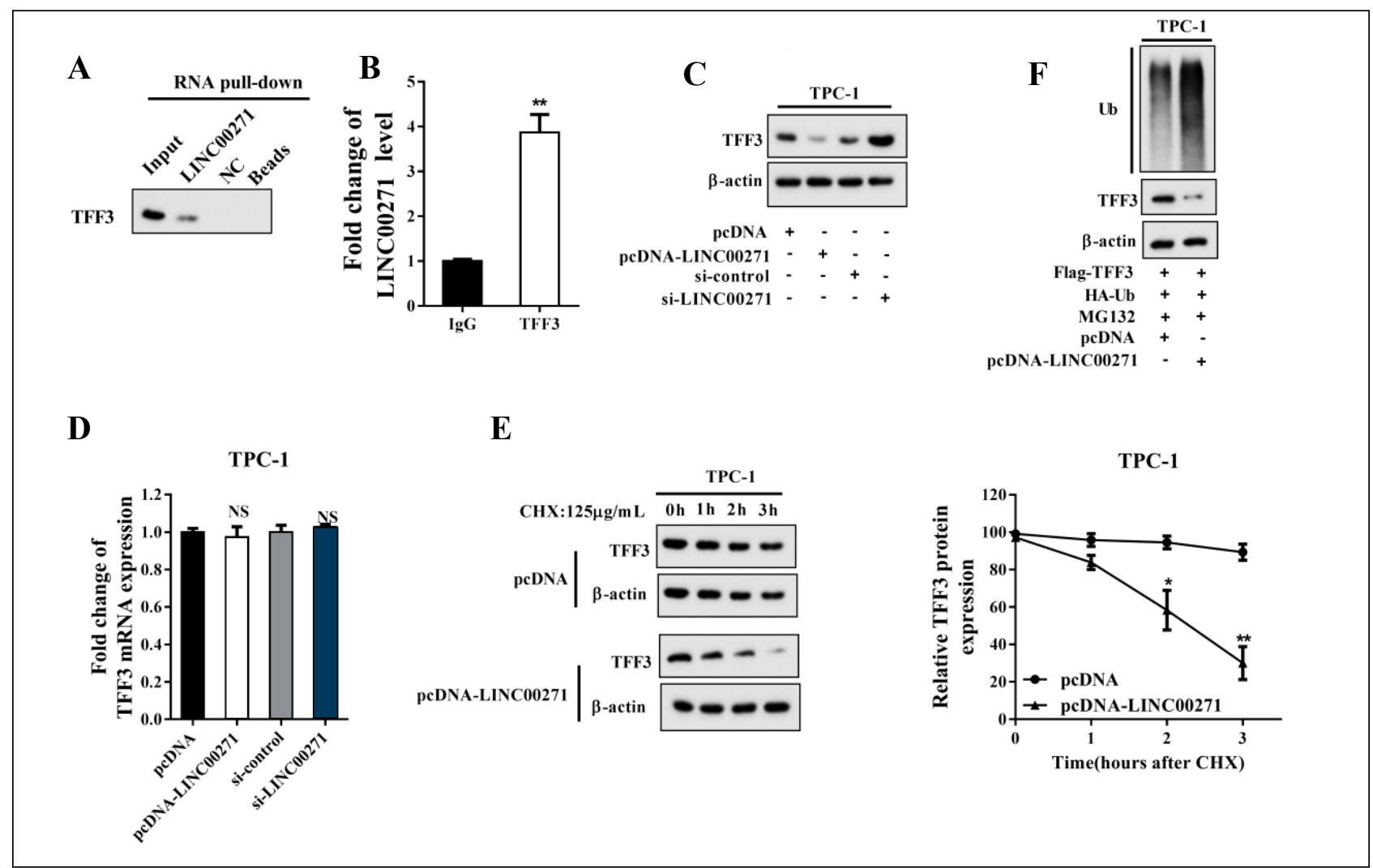

Figure 3. LINC00271 reduced the expression of TFF3 by increasing its ubiquitination. A: Detection of TFF3 using western blot in samples pulled down by biotinylated LINC00271; B: Combination of LINC00271 and TFF3 measured by RNA immunoprecipitation assay (RIP) and qRT-PCR; C: protein level of TFF3 measured by western blot after overexpressing or silencing LINC00271 in TPC-1 cells; D: mRNA level of TFF3 measured by qRT-PCR after overexpressing or silencing LINC00271 in TPC-1 cells; E: after overexpressing LINC00271, TFF3 expression measured by western blot $0,1,2$, and $3 \mathrm{~h}$ after incubation of TPC-1 cells with CHX $(125 \mu \mathrm{g} / \mathrm{ml})$. Beta-actin was used as an internal control. ImageJ was used to quantify TFF3 band densitometry. F: Ubiquitination (Ub) level of TFF3 measured by immunoprecipitation and western blot after overexpressing LINC00271 and treating TPC-1 cells with MG132 $(10 \mathrm{nM})$ for $8 \mathrm{~h}$. $* \mathrm{P}<0.05 \mathrm{vs}$. pcDNA, $* * \mathrm{P}<0.01 \mathrm{vs}$. IgG or pcDNA. 


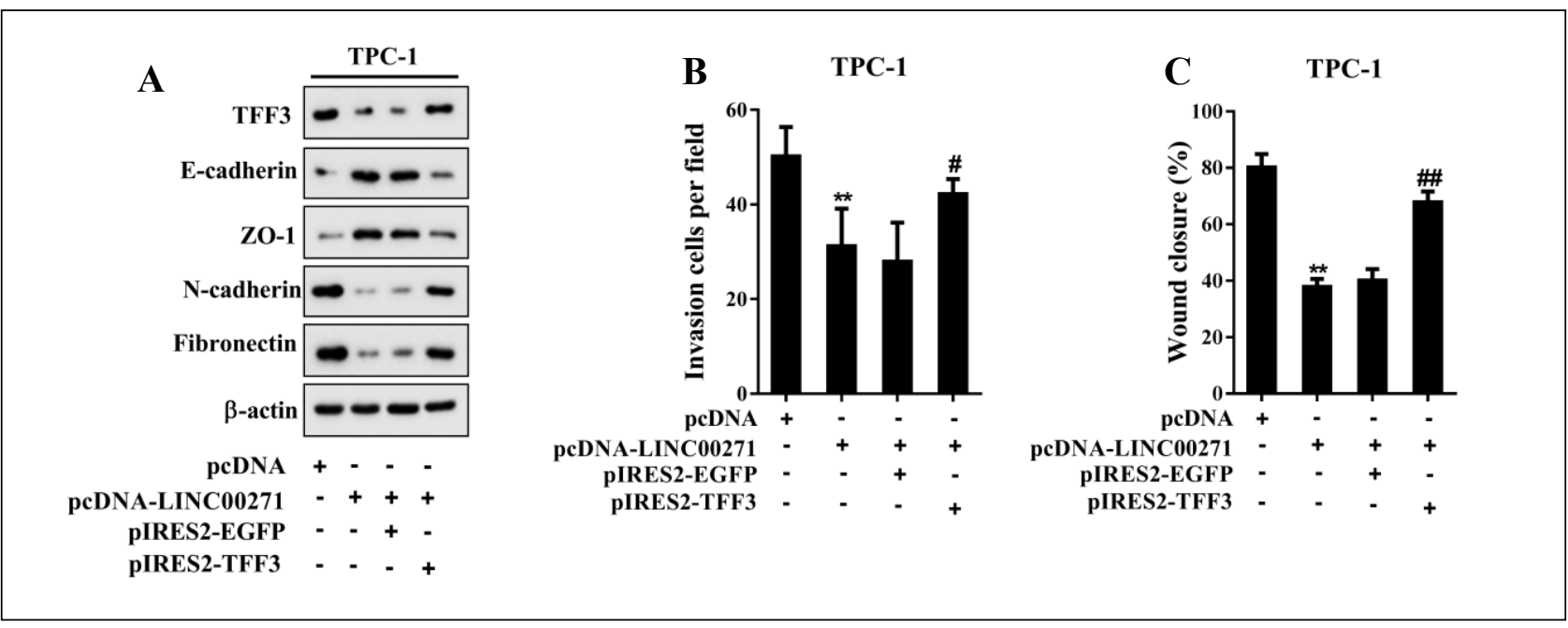

Figure 4. LINC00271 inhibited the EMT of PTC cells by targeting TFF3. TPC-1 cells were divided into four groups: pcDNA, pcDNA-LINC00271, pcDNA-LINC00271 + pIRES2-EGFP, and pcDNA-LINC00271 + pIRES2-TFF3. A: Protein levels of TFF3, E-cadherin, ZO-1, N-cadherin, and fibronectin measured by western blot. Beta-actin was used as an internal control. B: Invasive capability of TPC-1 cells determined by a Transwell invasion assay; $\mathbf{C}$ : migratory capability of TPC- 1 cells determined by a wound-healing assay. ${ }^{*} * \mathrm{P}<0.01 \mathrm{vs}$. pcDNA, \# $\mathrm{P}<0.05$ vs. pcDNALINC00271 + pIRES2-EGFP, \#\# P $<0.01$ vs. pcDNA-LINC00271 + pIRES2-EGFP.

neither pcDNA-LINC00271 nor si-LINC00271 affected the mRNA level of TFF3 (Figure 3D). To further explore whether LINC00271 downregulated the expression of TFF3 by affecting its stability, we blocked protein synthesis in TPC-1 cells using protein synthesis inhibitor CHX $(125 \mu \mathrm{g} / \mathrm{ml})$ and measured the TFF3 protein levels $0,1,2$, and $3 \mathrm{~h}$ after $\mathrm{CHX}$ treatment. Figure $3 \mathrm{E}$ shows that pcDNA-LINC00271 continuously decreased TFF3 expression in comparison with pcDNA. A further ubiquitination assay revealed that LINC00271 suppressed the expression of TFF3 by increasing its ubiquitination level (Figure 3F).

\section{LINC00271 inhibited the EMT of PTC cells by target- ing TFF3}

To further investigate whether TFF3 was the functional target of LINC00271 for inhibiting the EMT of PTC cells, we conducted a series of follow-up experiments. We overexpressed LINC00271 in TPC-1 cells by pcDNALINC00271 transfection and overexpressed LINC00271 and TFF3 by pcDNA-LINC00271 + pIRES2-TFF3 cotransfection. As shown in Figure 4A, LINC00271 overexpression reduced the expression levels of mesenchymal markers (N-cadherin and fibronectin) and increased those of epithelial markers (E-cadherin and ZO-1), while this trend was reversed by TFF3 upregulation. Moreover, Transwell invasion (Figure 4B) and wound-healing assays (Figure 4C) revealed that the decrease in the invasive and migratory capabilities of TPC-1 cells associated with LINC00271 overexpression was attenuated by TFF3 overexpression. These findings suggested that LINC00271 exerted its inhibitory effect on the EMT of TPC-1 cells by inhibiting TFF3 expression.

\section{Discussion}

Metastasis of PTC is the most important factor in the prognosis of the disease. As an essential promoter of cancer cell invasion and migration, the significance of EMT in PTC metastasis is undoubted [3]. In this study, we investigated the role of LINC00271 in PTC and found that LINC00271 prevented the EMT of PTC cells via downregulating TFF3 expression.

Previous studies have reported downregulation of LINC00271 in several cancers, such as oral squamous cell carcinoma, adrenocortical carcinoma, and thyroid cancer [18-20]. Its low expression is associated with poor prognosis, indicating that it may serve as a tumor suppressor gene. In this study, we found low expression levels of LINC00271 in both tumor tissue of PTC patients and a PTC cell line. LINC00271 overexpression significantly reduced the expression of $\mathrm{N}$-cadherin and fibronectin while increasing the expression of E-cadherin and ZO-1. N-cadherin and fibronectin are mesenchymal markers, whereas E-cadherin and ZO-1 are epithelial cell markers [21]. It has been reported that a decrease in epithelial marker levels and an increase in mesenchymal marker levels are decisive events of EMT that contribute to cancer metastasis [22]. Our results showed that LINC00271 overexpression prevented the EMT of PTC cells. Our in vivo study revealed that overexpressed LINC00271 reduced the tumor volume in xenograft model mice, confirming its antitumor effect in PTC.

It is noteworthy that in our study, TFF3 expression in tumor tissue of PTC patients inversely correlated with the expression of LINC00271. LINC00271 overexpression reduced the TFF3 protein level, while the knockdown of LINC00271 increased it. In contrast, neither pcDNALINC00271 nor si-LINC00271 affected the mRNA level of TFF3. Previous studies have shown that s regulate the expression levels of proteins by affecting their stability $[23,24]$. In light of this finding, we explored whether 
LINC00271 reduced TFF3 expression under the same mechanism. Indeed, CHX-chase and ubiquitination assays showed that LINC00271 overexpression elevated the ubiquitination level of TFF3, thus accelerating its degradation and reducing its protein level.

High expression of TFF3 has been reported to promote EMT in PTC progression [8]. Moreover, Batlle et al. reported that TFF3 reduces E-cadherin expression by repressing its transcription [25]. In line with previous studies, we found that TFF3 overexpression reduced the expression of epithelial cell markers and elevated the expression of mesenchymal markers in TPC-1 cells transfected with pcDNA- LINC00271. These findings suggest that TFF3 mediates the regulatory effect of LINC00271 on the EMT of PTC cells.

In conclusion, our study demonstrated the antitumor effect of LINC00271 on PTC and elucidated its regulatory mechanism in the EMT of PTC cells, offering a new prospect for the prevention of PTC metastasis.

\section{Declarations}

Conflict of interest: The authors declare no conflict of interest.

Authors' contributions: Jianda Dong: Conceptualization. Pihong Li and Xiaoyu Pan: Data curation, Writing- Original draft preparation.

Zhouci Zheng: Partial animal experiments.

Yihan Sun: Data analysis.

Yifan Han: Complete examination of the manuscript.

\section{References}

1. Fagin J A, Wells Jr S A. Biologic and clinical perspectives on thyroid cancer. New England Journal of Medicine, 2016, 375(11): 1054-1067.

2. Grubbs E G, Evans D B. Role of lymph node dissection in primary surgery for thyroid cancer. Journal of the National Comprehensive Cancer Network, 2007, 5(6): 623630.

3. Shakib H, Rajabi S, Dehghan M H, et al. Epithelial-to-mesenchymal transition in thyroid cancer: a comprehensive review. Endocrine, 2019: 1-21.

4. May F E B, Westley B R. TFF3 is a valuable predictive biomarker of endocrine response in metastatic breast cancer. Endocrine-related cancer, 2015, 22(3): 465.

5. Kannan N, Kang J, Kong X, et al. Trefoil factor 3 is oncogenic and mediates anti-estrogen resistance in human mammary carcinoma. Neoplasia (New York, NY), 2010, 12(12): 1041.

6. Pandey V, Zhang M, You M, et al. Expression of two nonmutated genetic elements is sufficient to stimulate oncogenic transformation of human mammary epithelial cells. Cell death \& disease, 2018, 9(12): 1-18.

7. Krause K, Eszlinger M, Gimm 0, et al. TFF3-based candi- date gene discrimination of benign and malignant thyroid tumors in a region with borderline iodine deficiency. The Journal of Clinical Endocrinology \& Metabolism, 2008, 93(4): 1390-1393.

8. Lin X, Zhang H, Dai J, et al. TFF3 Contributes to Epithelial-Mesenchymal Transition (EMT) in papillary thyroid carcinoma cells via the MAPK/ERK signaling pathway. Journal of Cancer, 2018, 9(23): 4430.

9. Xia L, Wu L, Bao J, et al. Circular RNA circ-CBFB promotes proliferation and inhibits apoptosis in chronic lymphocytic leukemia through regulating miR-607/FZD3/ Wnt/ $\beta$-catenin pathway. Biochemical and biophysical research communications, 2018, 503(1): 385-390.

10. Xiong X, Ren X, Cai M, et al. Long non-coding RNAs: An emerging powerhouse in the battle between life and death of tumor cells. Drug Resistance Updates, 2016, 26: 28-42.

11. Ma B, Liao $T$, Wen $D$, et al. Long intergenic non-coding RNA 271 is predictive of a poorer prognosis of papillary thyroid cancer. Scientific reports, 2016, 6(1): 1-13.

12. Yang Z, Yu W, Huang R, et al. SIRT6/HIF- $1 \alpha$ axis promotes papillary thyroid cancer progression by inducing epithelial-mesenchymal transition. Cancer cell international, 2019, 19(1): 17.

13. Shi M, Hao J, Wang X W, et al. Functional Dissection of pri-miR-290 295 in Dgcr8 Knockout Mouse Embryonic Stem Cells. International journal of molecular sciences, 2019, 20(18): 4345.

14. Huang J Z, Chen M, Zeng M, et al. Down-regulation of TRPS1 stimulates epithelial-mesenchymal transition and metastasis through repression of FOXA1. The Journal of pathology, 2016, 239(2): 186-196.

15. Baiz D, Dapas B, Farra R, et al. Bortezomib effect on E2F and cyclin family members in human hepatocellular carcinoma cell lines. World journal of gastroenterology: WJG, 2014, 20(3): 795.

16. Kong G, Jiang Y, Sun X, et al. Irisin reverses the IL-6 induced epithelial-mesenchymal transition in osteosarcoma cell migration and invasion through the STAT3/Snail signaling pathway. Oncology reports, 2017, 38(5): 26472656.

17. Zhu Y, Tan J, Xie H, et al. HIF-1 $\alpha$ regulates EMT via the Snail and $\beta$-catenin pathways in paraquat poisoninginduced early pulmonary fibrosis. Journal of cellular and molecular medicine, 2016, 20(4): 688-697.

18. Zhou S, Zhu Y, He Z, et al. Long Non-Coding RNA Expression Profile Associated with Malignant Progression of Oral Submucous Fibrosis. Journal of oncology, 2019, 2019.

19. Buishand F O, Liu-Chittenden Y, Fan Y, et al. Adrenocortical tumors have a distinct, long, non-coding RNA expression profile and LINC00271 is downregulated in malignancy. Surgery, 2020, 167(1): 224-232.

20. Murugan A K, Munirajan A K, Alzahrani A S. Long noncoding RNAs: emerging players in thyroid cancer pathogenesis. Endocrine-related cancer, 2018, 25(2): R59R82.

21. Paolillo M, Schinelli S. Extracellular Matrix Alterations in 
Metastatic Processes. International journal of molecular sciences, 2019, 20(19): 4947.

22. Lu W, Kang Y. Epithelial-mesenchymal plasticity in cancer progression and metastasis. Developmental cell, 2019, 49(3): 361-374.

23. Yang F, Huo X, Yuan S, et al. Repression of the long noncoding RNA-LET by histone deacetylase 3 contributes to hypoxia-mediated metastasis. Molecular cell, 2013, 49(6): 1083-1096.
24. Li Z, Hou P, Fan D, et al. The degradation of EZH2 mediated by IncRNA ANCR attenuated the invasion and metastasis of breast cancer. Cell Death \& Differentiation, 2017, 24(1): 59-71.

25. zum Büschenfelde D M, Hoschützky $H$, Tauber R, et al. Molecular mechanisms involved in TFF3 peptide-mediated modulation of the E-cadherin/catenin cell adhesion complex. Peptides, 2004, 25(5): 873-883.

Cite this article as: $\mathrm{Li} \mathrm{P}, \mathrm{Pan} \mathrm{X}$, Zheng Z, et al. LINC00271 inhibits epithelial-mesenchymal transition of papillary thyroid cancer cells by downregulating trefoil factor 3 expression[J]. Aging Pathobiology and Therapeutics, 2020, 2(2): 78-85. 